\title{
The challenge of caring for patients with rare diseases
}

One of the greatest challenges a physician is likely to face in their career is caring for the patient with a rare disease. While medical education now encompasses an often-bewildering range of subjects, the core principles of how medical students are taught to recognize disease remains the same: repetition and pattern recognition. For any set of symptoms, learners are taught to recognize the responsible afflictions that are the most common. As the old proverb goes: "When you hear hoofbeats, think of horses and not zebras".

Yet what happens when it is indeed the "zebra" that we are looking for? This is the challenge of recognizing and treating rare diseases. In this issue, we present three cases of rare diseases encountered in the hospital. First, a 40-year-old woman receives in vitro fertilization treatment but subsequently develops portal vein thrombosis, a complication described only once before in the literature. Next, a toddler with overgrowth syndrome presents to hospital with decreased level of consciousness, whereupon genetic testing revealed a rare de novo mutation consistent with 19p13.13 Microdeletion Syndrome. Finally, two cases of elderly men diagnosed with plasmacytoid urothelial carcinoma are described, detailing the struggle physicians faced in managing this rare and aggressive bladder cancer variant.

Too often, patients with rare diseases are not properly diagnosed and consequently do not receive proper care. Due to their rarity of these diseases, and the consequent scarcity of published studies on them, it can be extremely challenging for physicians to be able to diagnose and manage these cases with confidence. Case reports like the ones presented in this issue are therefore a valuable tool for physicians to develop awareness of rare diseases and be better prepared to recognize and manage any such "zebras" that may cross their path.

Charles Yin

Senior Associate Editor 